\title{
Stakeholders y outcomes no intencionados en la
}

comunicación estratégica

\section{Stakeholders and unintended outcomes in strategic communication}

\section{Stakeholders e outcomes não intencionados na comunicação estratégica}

DOI: https://doi.org/10.18861/ic.2020.15.1.2957

FRANCISCO L. LÓPEZ DELCASTILLO WILDERBEEK

franciscoleslie@alumni.upf.edu - Universitat Pompeu Fabra, España.

ORCID: https://orcid.org/0000-0002-6664-7849

Fecha de recepción: 15 de enero de 2020

Fecha de aceptación: 21 de abril de 2020

\section{RESUMEN}

Los resultados no deseados de las acciones de comunicación se conocen como outcomes no intencionados según los modelos de evaluación más extendidos. La medición de estos outcomes adquiere enorme relevancia en tanto pueden representar efectos adversos para los grupos de interés (stakeholders) que están vincula- dos a la organización: esta correlación pone en peligro los objetivos comunicativos y puede impactar negativamente en la actividad. En este artículo se analiza el resultado no deseado de una acción de comunicación estratégica. De manera más específica, se propone un análisis de contenido de la aparición de críticas en los medios a las políticas engañosas sobre compromiso medioambiental (greenwashing) durantela celebración de la Conferencia de las Naciones Unidas sobre Cambio Climático (COP25) realizada en Madrid en 2019. A través de la observación cuantitativa de la cobertura mediática y la cuantificación de las variables extraídas, se llega a definir la verdadera implicación mediática de la perspectiva del greenwashing y, específicamente, su afectación en los potenciales stakeholders.

PALABRAS CLAVE: stakeholders, outcomes no intencionados, evaluación, comunicación estratégica, relaciones públicas, greenwashing. 


\section{ABSTRACT}

The uncontrolled reactions caused by communication actions are known as outcomes unintended according to the most widespread evaluation models. The measurement of these outcomes becomes enormously relevant as they can represent adverse effects for the stakeholders that arelinked to the organization: this correlation jeopardizes communication goals and could impact its activity in a negative way. This article discusses the unwanted outcome of a strategic communication action. More specifically, a content analysis is carried out of the appearance of criticisms of misleading policies on environmental commitment (greenwashing) that occurred in the framework of the Climate Summit (COP25) held in Madrid in December 2019. Through จั the quantitative observation of the media coverage ․ and the quantification of the extracted variables, it is possible to define the true media involvement of the ? greenwashing perspective, and, specifically, its effecton potential stakeholders.

134 KEYWORDS: stakeholders, unintended outcomes, evalua㟧 tion, strategic communication, public relations, green-

\section{RESUMO}

Reações não controladas geradas por ações de comunicaçãosão conhecidascomo outcomes, nãointencionados de acordo com os modelos deavaliação mais difundidos. A mensuração desses outcomestorna-se extremamente relevante, pois podem representar efeitos adversos para os grupos de interesse (stakeholders) que estão ligados à organização: essa correlação põe em risco os objetivos da comunicação e pode impactar a organização negativamente na atividade. Este artigo analisa o resultado indesejado de uma ação de comunicação estratégica. Mais especificamente, uma análise de conteúdo é realizada a partir do surgimento de críticas a políticas enganosas sobre compromisso ambiental (greenwashing) que ocorreramnocontexto da Cúpula doClima(COP25), realizada em Madri em dezembro de 2019. Através da observação quantitativa da cobertura da mídia e da quantificação das variáveis extraídas, é possível definir a verdadeira implicação da mídia na perspectiva da greenwashing e, especificamente, seu efeito sobrepotenciaisstakeholders.

PALAVRAS-CHAVE: stakeholders, outcomes não intencionados, avaliação, comunicação estratégica, relações públicas, greenwashing. 


\section{INTRODUCCIÓN}

La comunicación estratégica representa un concepto amplio basado en "cómo las organizaciones usan la comunicación a través de disciplinas profesionales tradicionales para alcanzar las metas y los objetivos marcados" (Hallahan, 2013, p. 161) desde una perspectiva global y estratégica (Argenti, Howell \& Beck, 2005).

Las relaciones públicas son uno de estos ámbitos profesionales, su misión es "establecer y mantener relaciones mutuamente beneficiosas con los grupos de interés clave" (Hallahan, Holtzhausen, Van Ruler, Verčič \& Sriramesh, 2007, p.6). Estos grupos de interés se conocen como stakeholders y representan un factor determinante en la actividad de las organizaciones (Freeman \& Reed, 1983). No en vano es usual en el campo de las relaciones públicas que el concepto de stakeholder sea intercambiable con el de público (Pieczka, 2019) ya que "la planificación y la gestión de las relaciones públicas de una organización está condicionada por los intereses de cada público" (Capriotti, 2007, p.77)1. Como indican Macnamaray Gregory (2018) un "cuerpo emergente de literatura sobre comunicación estratégica insta a prestar atención tanto a los intereses de los stakeholders como a los intereses propios de la organización" (p. 772).

En este sentido, los medios de comunicación forman parte de los grupos de interés al estar dotados de capacidad de influencia suficiente para que las organizaciones alcancen o no sus objetivos, especialmente en los momentos de crisis (Rawlins, 2006). De esta forma, los medios de comunicación se articulan como un público trascendente para la consecución delos objetivos establecidos en la comunicación estratégica.

\section{MARCO TEÓRICO}

\subsection{Evaluación de la comunicación estratégica}

Pese a que es posible encontrar una base importante de modelos teóricos de evaluación aplicables en la comunicación estratégica (Macnamara, 2018c), no hay unanimidad académica sobre los indicadores de medición y evaluación más adecuados para confirmar el cumplimiento de los objetivos estratégicos (Madroñero \& Capriotti, 2018). El trabajo de Macnamara y Gregory (2018), basado en entrevistas en profundidad y análisis de contenido, se centró en los seis modelos de evaluación de comunicación estratégica más extendidos. Sus conclusiones indicaron que tres de estos modelos inciden en la importancia de los objetivos para el alineamiento de la acción y la evaluación de la comunicación: The European Commission Directorate-General ofCommunicationsmodel of evaluation, UK Government Communication Service Model y finalmente The

1 Castillo Esparcia (2010) evidencia esta importancia cuando insiste en que la traducción correcta de la acepción inglesa debería ser "relaciones con los públicos". 
AMEC Integrated Evaluation Framework (AMEC), que coloca objetivos en el primer lugar para luego desarrollar el plan de acción.

En todos los modelos analizados se detectó una focalización en los outcomes que pueden definirse como cambios en las opiniones, actitudes o comportamiento de los públicos. Michaelson y Stacks (2011) señalan, desde una perspectiva general, que los outcomes pueden entenderse como la materialización perceptible de la fidelidad de la audiencia hacia una marca, producto, servicio o acontecimiento.

Los outcomes forman parte delos modelos deevaluación dela comunicación estratégica entendidos como una cadena secuencial que incluye los outputs, los outtakes y el impacto o resultado final. Los outputs miden el nivel de atención o cobertura que ha logrado una acción de las relaciones públicas (Lindenmann, 1997) $)^{2}$, los outtakes suponen un dato intermedio entre outputs y outcomes que sirve de indicio sobre los efectos que van a obtenerse. Un ejemplo serían los datos obtenidos desde plataformas digitales como número de descargas de un determinado contenido o la cantidad de comentarios realizados en social media (Macnamara,2014). Ahora bien, la acumulación de estos elementos no es un fin en sí mismo para el análisis de las relaciones públicas y puede conducir a lo que se ha dado en llamar "métricas de la vanidad" (vanitymetrics) (Bartholomew citadoen Macnamara, 2018a). Por otrolado, el impacto (Macnamara \& Gregory, 2018) o resultado final representa la traducción delos esfuerzos delas relaciones públicas y de la comunicación estratégica de forma que afecten positivamente a la organización desde una perspectiva comercial o de negocio.

Diagrama 1. Marco de evaluación de la AMEC

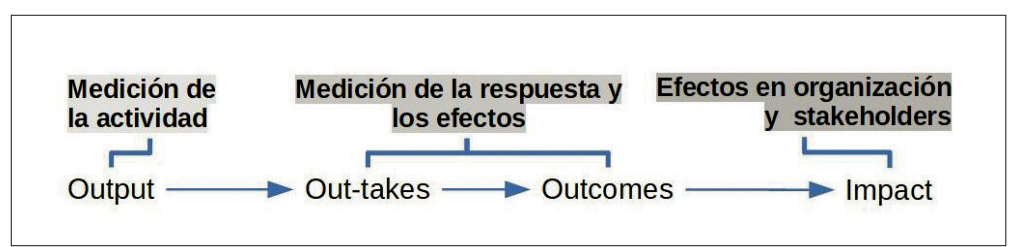

Fuente: Elaboración propia con datos de la AMEC.

En la actualidad, la industria dedicada al análisis de las acciones de comunicación apuesta por la evaluación de los resultados efectivos (outcomes) ${ }^{3}$. Esto es debido a que son variables que van más allá de las coberturas logradas en los medios y buscan datos que sean reflejo de los resultados obtenidos y de la verdadera influencia de estas acciones. A su vez este ejercicio de transparencia

2 Como indica Place (2015), algunas definiciones más específicas a nivel operativo extraen los inputs como la comunicación generada y distribuida por los profesionales de las relaciones públicas. De esta forma los outputs quedarían sólo como la cobertura alcanzada por esas comunicaciones.

3 Especialmente, desde la implantación de los Barcelona Principles en 2010 (Schriner, Swenson \& Gilkerson, 2017; Watson \& Noble, 2014). 
puede traducirse en una interpretación positiva por parte de los grupos de interés relacionados con cada organización (Place, 2015).

\subsection{Outcomes no intencionados}

Como se ha comentado anteriormente los outcomes, entendidos como resultados de las acciones de comunicación y, específicamente, de las relaciones públicas, son especialmente importantes para la medición del cumplimiento de los objetivos de esta actividad. En este sentido se refiere Xifra (2007) cuando indica que los objetivos de relaciones públicas deben ser cuantificables y deben estar conectados con un propósito determinado. Los outcomes representan el detalle de esa variable medible que otorga validez a la tasa de éxito de los objetivos de la comunicación.

A este respecto, Madroñero y Capriotti (2018) constataron que las lagunas en los indicadores para evaluar las relaciones públicas pueden ser debidas a la inconcreción sobre los objetivos estratégicos, carencia que se torna significativa en relación a la observación de los outcomes (Buhmann \& Brønn, 2018; Söderqvist, 2017). Semejante vacío puede deberse a la opacidad de las organizaciones que aplican algún tipo de metodología en la evaluación de la comunicación (Schriner, Swenson \& Gilkerson, 2017; Swensonet al., 2019), o especialmente a la dificultad relacionada con la medición de los outcomes en comparación con las tasas de cobertura lograda y equivalencias ${ }^{4}$ (Austin \& Pinkleton, 2015; l'Etang \& Pieczka, 2006; Lindenmann, 2003; Watson, 2013). La reconocida complejidad en la evaluación de los outcomes, pese a su vinculación con los objetivos de las acciones de comunicación, se magnifica especialmente cuando la variable que debe ser medida se considera un resultado (outcome) no intencionado o directamente no deseado.

Las acciones de comunicación de una organización movilizadas para el cumplimiento de ciertos objetivos pueden generar un efecto "negativo para los stakeholders, los públicos o la sociedad” (Macnamara \& Gregory, 2018, p. 483), en tal sentido "la comunicación estratégica debeimplicarlaevaluación desus resultados (outcomes) y del impacto de forma más amplia que los objetivos pretendidos e inmediatos de una organización" (ídem). Por lo tanto, el proceso de evaluación de las relaciones públicas dentro de la comunicación estratégica ha de ser capaz de dar cuenta de las consecuencias inesperadas y lesivas para las organizaciones, intentando, en la medida de lo posible, medir su alcance para que los profesionales apliquen un cambio o ejecuten nuevas acciones (Macnamara, 2017) ${ }^{\mathbf{5}}$ que les permitan alcanzar sus objetivos estratégicos (Hallahan et al., 2007).

4 Como por ejemplo el Advertising Value Equivalency.

5 Como indica Macnamara (2017), la mayoría de los modelos de evaluación no incluyen la predisposición a cambiar la comunicación estratégica según las actitudes observadas en los stakeholders. Una excepción sería el marco de análisis que aplica el gobierno de Nueva Gales del Sur (NWS) en el que se reconoce un flujo bidireccional entre organización y grupos de interés (véase: https://communications.dpc.nsw.gov.au/advertising/advertising requirements/evaluation-framework/). 


\subsection{Celebración de la COP25 en Madrid}

Del 2 al 13 de diciembre de 2019 la ciudad de Madrid acogió la Conferencia de las Naciones Unidas sobre Cambio Climático conocida como COP25. Esta cumbre recibió el patrocinio de diversas empresas por mediación del gobierno español que llegaron a invertir entre 10 y 12 millones de euros (Medina, 1 de diciembre de 2019). Las empresas patrocinadoras obtuvieron presencia relevante durante la Conferencia que, por un lado, se tradujo en cobertura de prensa favorable (publicity) ${ }^{\mathbf{6}} \mathrm{y}$, por otro, justificó que generaran publicidad nativa ${ }^{7}$ y patrocinio en los medios vinculado con la celebración de la cumbre.

El ejemplo más característico se produjo cuando la compañía energética Endesa (en la máxima categoría de patrocinio de la Conferencia) contrató como publicidad nativa a las portadas de 25 cabeceras de prensa para informar sobre la inversión en energía renovable de su nuevo plan estratégico. Respecto del patrocinio de secciones, la empresa energética Iberdrola (también en la máxima categoría de patrocinio) contrató la presencia de su logo en todas las noticias publicadas sobre la cumbre en el diario El País.

\subsection{El fenómeno greenwashing}

El greenwashing representa un concepto que se define como la aplicación superficial y engañosa de políticas medioambientales por parte de las organizaciones (Testa, Boiral \& Iraldo, 2018) y, en tal sentido, se lo considera un reverso negativo de la Responsabilidad Social Corporativa (Delmas \& Burbano, $2011)^{9}$. La revisión bibliográfica de Jong, Harkink y Barth (2018) concluyó que las dos características definitorias de este fenómeno residen en el distanciamiento respecto de la realidad (elemento intrínseco) y el uso de técnicas para confundir a las audiencias (elemento comunicativo). Por lo tanto, la cuestión esencial en este tipo de política se basa en la elaboración de una estrategia de comunicación que sea capaz de exagerar positivamente el punto de vista de las audiencias sobre el comportamiento ambiental de una organización (Lyon \& Montgomery, 2015) $)^{\mathbf{1 0}}$.

La promoción, por otra parte, de los esfuerzos medioambientales de las compañías queda bajo el riesgo de que los diferentes públicos consideren a estas acciones de comunicación como verdaderas distracciones que desvían la atención sobre las prácticas dela organización en un sentido global(Gallicano, 2011). Ante

6 Promoción aparecida en los medios que no corresponde a una contraprestación económica.

7 Publicidad con contenido informativo y formato coincidente con el medio en el que es incorporado.

8 Diario de prensa diaria español con más lectores según datos del Estudio General de Medios (EGM), España.

9 Estos autores diferencian entre el greenwashing a nivel de producto y el greenwashing a nivel corporativo.

10 Estos autores detallan, mediante una revisión bibliográfica, las prácticas más comunes de greenwashing por parte de las organizaciones: la revelación selectiva delas actividades que son no contaminantes, la elaboración de propuestas ambientales sin acción, la certificación ambiental poco rigurosa, la colaboración con entidades ecologistas, la par ticipación en campañas oficiales sin cumplir los objetivos establecidos y la elaboración de un discurso ecologista. 
este supuesto existela constatación empírica segúnla cualla detección delfenómeno de greenwashing afecta negativamente a la imagen de marca y a la voluntad de compra de los consumidores (Nyilasy, Gangadharbatla \& Paladino, 2014).

De esta forma, un enfoque centrado en los grupos de interés (Testa, Boiral \& Iraldo, 2018) advierte que tanto los accionistas como las empresas colaboradoras tienen la capacidad de forzar cambios reales en las compañías hacia negocios más sostenibles y ecológicos. Sin embargo, la influencia de clientes y de asociaciones del sector tienden a fomentar políticas de greenwashing de tal manera que se podría hablar de una dinámica retroalimentada. Las políticas de relaciones públicas orientadas a potenciar el posicionamiento medioambiental de la organización deberían discriminar entre los públicos legitimados para actuar directamente sobre sus políticas y los que no lo están. Por ejemplo, los accionistas son realmente los dueños de las compañías y esta íntima vinculación se observa en el mismo lenguaje empresarial con la máxima de "generar valor para el accionista" (Smith, 2003, p. 89).

A este respecto se refieren Scholes y Clutterbuck (1998) cuando advierten que los equipos financieros tienen cierta responsabilidad en la gestión de la comunicación con los inversores, mientras que la relación con los medios de comunicación que trasladarán los mensajes es territorio en exclusiva de los equipos de relaciones públicas.

\section{METODOLOGÍA}

Esta investigación se ha basado en el análisis de contenido como metodología para la observación del discurso mediático en prensa escrita, prensa online y blogs en España sobre la Conferencia sobre Cambio Climático (COP25). El análisis de contenido es una "técnica de investigación para la elaboración de inferencias válidas y replicables” (Krippendorff, 2018, p.403) de las características de un mensaje, de una manera sistemática, objetiva y cuantitativa (Neuendorf, 2017). Un elemento clave para dar validez a esta metodología es que su aplicación es independiente del analista y del material analizado, y evita por tanto interpretaciones ambiguas (Krippendorff, 2018).

Como aporta Mcnamara (2018b), la técnica más habitual para categorizar los elementos detectados mediante el análisis de contenido en medios de comunicación es la aplicación de codificación de las características como ser la presencia de palabras clave determinadas en cada contenido. Este autor comenta la existencia de tres variantes de codificación: mediante el trabajo de un analista, mediante la recuperación de información usando palabras clave en una base de datos y, finalmente, mediante la aplicación de Procesamiento de Lenguaje Natural, modelo en que el analista pierde casi totalmente la capacidad de supervisión sobre el corpus.

En estainvestigación se aplicaron los modelos primeroysegundo, yseaprovecharonlastécnicas derecuperación deinformación sehan extraídolos contenidos 
de prensa y de prensa online publicados en España en que se menciona la COP25. Algunas de las características de estos textos se han cuantificado e interpretado con el propósito de obtener una visión contrastada sobre el impacto mediático de la celebración de la COP25 en relación a un efecto no intencionado de esta misma exposición mediática: la consideración de prácticas de greenwashing.

Concretamente se ha tenido acceso a la base de datos de la empresa REBOLD, líder en el análisis de la comunicación mediante datos. Para la recuperación de información se tomó como universo todos los recortes de prensa escrita de todos los medios publicados en España, todas las noticias de prensa online de 2.951 portales de noticias en España y todos los posts de 9.537 blogs en el mismo país. El marco temporal incluyó de las 00:00 horas del 2 de diciembre a las 23:59 horas del 16 de diciembre ${ }^{11}$.

De este universo se extrajo información de todos los contenidos que incluían el término COP25 y todos los contenidos que incluían la combinación COP25 y greenwashing. El corpus resultante fue analizado tomando como variables relevantes la tipología del contenido (prensa escrita/online/blogs), la categorización del medio (nacional, regional, económico, nativo), los datos de audiencia, el rol desempeñado por el concepto greenwashing dentro del texto y otros elementos como la relación entre contenidos online y medios matriz (crossmedia) o los contenidos como réplica de teletipo de agencia de noticia.

Por otro lado, debido a la disparidad de audiencias en los medios digitales entre los medios más visitados y los menos visitados, para la determinación de la relación positiva o negativa del término greenwashing se aplicó una selección de webs según audiencia y participación en medios tradicionales. La selección se produjo analizando contenidos de los portales que fueran unidades digitales de un medio tradicional (prensa económica o nacional) o que estuvieran entre los diez medios nativos con más audiencia (Nativo T1) según datos de la empresa de medición en Internet Comscore ${ }^{12}$.

Como podrá verse en el apartado de resultados, el total de contenidos analizados sobre vinculación positiva o negativa fueron 23 respecto de 129 que quedaron descartados. Sin embargo, la ordenación mediante la variable de audiencia denotó que esos 23 portales (Nativo T1 y unidades digitales de medios tradicionales) alcanzaron a una audiencia potencial 11,5 veces mayor que el resto de los contenidos (Nativo T2) ${ }^{13}$. Es decir, el conjunto de audiencia impactada en la selección ( 23 medios) fue muy superior si se compara con el conjunto de medios no seleccionados (129 medios), pese a que estos hubieran generado más contenidos.

11 El marco temporal tomó como fecha de inicio la apertura de la cumbre y como fecha de cierre el lunes 16 de diciem bre, día en que la ministra de Transición Ecológica hizo un balance de la cumbre en la televisión pública española.

12 Véase: https://www.economiadigital.es/politica-y-sociedad/los-diez-periodicos-digitales-espanoles-mas-importantes-por-audiencia_570932_102.html (10 de enero de 2020).

13 Audiencia de 35.546.775 en nacionales, económicos y Nativo T1 con un total de 23 entradas (media de 1.545 .511 personas) / Audiencia de 17.378.592 y Nativo T2 con un total de 129 entradas (media de 134.717 personas). 


\section{RESULTADOS}

\subsection{Cobertura y exposición}

El marco metodológico descrito permitió una primera consulta de un total de 4.985 recortes de prensa, 18.693 noticias de prensa online y 864 posts de blogs. Todos estos contenidos incluían el término COP25. Como puede apreciarse en el Gráfico 1, que incluye prensa escrita y agrupa medios digitales (noticias y blogs), la evolución en la generación y publicación de contenidos fue de tipo lineal, exceptuando un volumen por encima de la media en la inauguración realizada los días 2 y 3 de diciembre (la repercusión en prensa diaria tradicional tuvo una desviación de 24 horas por la lógica de producción $)^{14}$ y un descenso en la producción de online coincidente con los días 8, 14, 15 y 16 de diciembre (días sin actos programados de la cumbre).

Gráfico 1. Gráfico cuantitativo en línea de tiempo con el total de contenidos publicados con la expresión COP25 dentro de la franja analizada y separada por prensa tradicional / medios online (noticias y blogs)

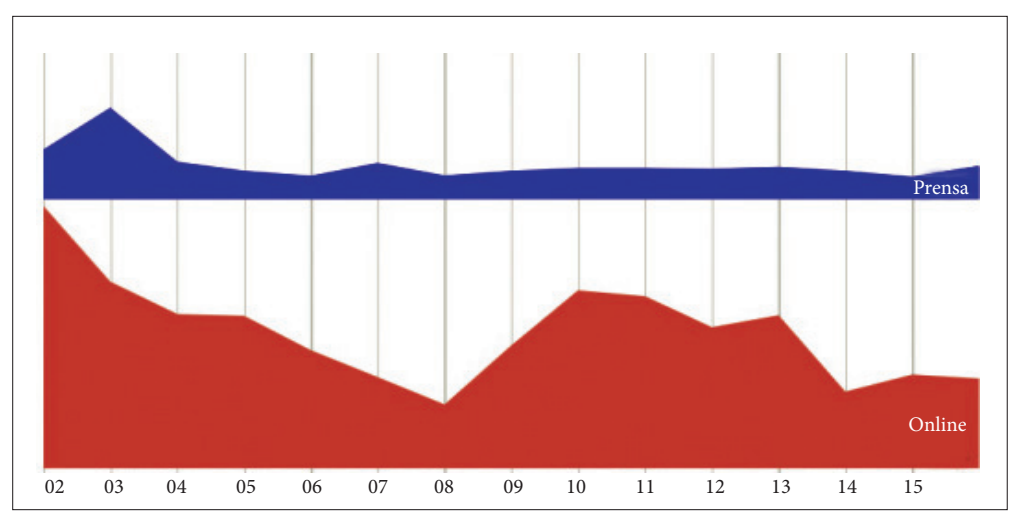

Fuente: elaboración propia con datos REBOLD.

Ahora bien, esta aproximación de tipo cuantitativo está referida a la cobertura que logró el acontecimiento tanto de forma independiente como a través de publicidad nativa pagada por las empresas patrocinadoras. Esta medición está circunscrita a la propia actividad de los profesionales de las relaciones públicas, dado que es un espacio en gran parte ganado en los medios por lo que debe considerarse outputs en la evaluación de la comunicación estratégica y no un resultado dentro de los objetivos establecidos.

Respecto al uso del término greenwashing circunscrito a la Conferencia este alcanzó un total de 35 artículos de prensa diaria tradicional, 142 contenidos de

14 Los medios digitales publican contenido de manera constante, mientras que la prensa gráfica publicada en papel publica una vez al día. 
prensa onliney 10 posts deblogs ${ }^{15}$. Delos 35 artículos de prensa se descartaron 9 cabeceras regionales del medio ElMundo, por ser contenidos idénticos dentro delas diferentesediciones regionalesquetambién se encontraron enlaedición nacional.

En términos de audiencia potencial ${ }^{16}$, el total de personas expuestas a los mensajes que citaron el fenómeno de greenwashing en relación a la cumbre fueron 52.925.367 en medios online $e^{17}$ y 5.113.072 lectores en prensa tradicional.

El elemento relevante en todo caso es la presencia de un concepto disonante como es la utilización del término greenwashing en los contenidos relacionados con la cumbre. En este escenario se puede hablar de un outcome no intencionado porque es un cambio involuntario en la línea narrativa promocionada en los medios siempre y cuando reciba un enfoque contrario a los intereses de la acción de comunicación estratégica.

Concretamente, el medio El Confidencial (Nativo T1) alertó que "los principales patrocinadores han sido acusados de aprovechar la cita para hacer", es decir, pagar para asociar su marca a un evento 'verde' mientras siguen adelante con intereses empresariales" (Villarreal, 3 de diciembre de 2019). Por lo tanto, existe un objetivo conativo sustentado en la exposición de cobertura positiva que busca un cambio en la actitud de la audiencia (outcome intencionado) y que podría ser medido, por ejemplo, con encuestas ex-ante y ex-post. Este propósito, sin embargo, hace que se manifieste un nexo causal que vincula por un lado el outcome potencial pretendido (mejorar la imagen medioambiental de las empresas organizadoras) con una línea argumental negativa no intencionada. La diferencia entre el efecto buscado - que se encuentra en grado de tentativa- y la evidencia de un resultado contrario (outcome no intencionado) reside en el cambio de comportamiento de los medios como stakeholders de las organizaciones. Dentrodeun flujo de contenidos promovidos desdediferentesfrentes (pagadosy no pagados), algunos medios expresan un cambio en la corriente dominante, reconociendo una relación de causa y efecto respecto esa misma cobertura positiva.

Ambas dimensiones son indisolubles y por ello ambas deben ser evaluadas dentro de una concepción estratégica dela comunicación. Este trabajo sehacentrado en la vertiente involuntaria precisamente porque se evidencia su posición de outcome negativo: representa un resultado perjudicial para la comunicación estratégica y un posible cambio en el punto de vista expresado por los medios como grupos de interés que influyen de forma relevante en las organizaciones.

Tomando una perspectiva meramente cuantitativa puede observarse que el conjunto de contenidos que formaron parte de este outcome no intencionado fue claramente inferior respecto el volumen mediáticogenerado porla COP25 en una cifra aproximada al $1 \%$ respecto al total de contenidos (tanto en prensa como en

15 Sin importar la relación entre el concepto y la celebración de la Conferencia sobre Cambio Climático.

16 Tomando como referencia el total de personas que podrían haber accedido al contenido según las audiencias estándar de los medios en las fechas analizadas, sin desagregar las personas que pueden haber tenido acceso al mensaje más de una vez durante la franja.

17127.143 en blogs y 52.798 .224 en prensa online. 
digital). Sin embargo, su sentido presumiblemente contradictorio ala celebración de la cumbre requiere de un análisis más en profundidad para valorar una posible reformulación de la comunicación estratégica de los actores involucrados.

\subsection{Análisis de la prensa online y blogs}

La aplicación del análisis de contenido en los medios de prensa online y blogs indicó, pese a la existencia de una exposición medida cuantitativamente, que no puede hablarse de una conexión directa entre el concepto peyorativo del maquillaje medioambiental respecto de la celebración de la COP25. Si bien de los 23 contenidos analizados 12 mostraron una vinculación negativa con el uso del término greenwashing, 3 no se computaron al tratarse de glosarios y 8 directamente fueron noticias de carácter positivo. La cosmética ecológica en estos 3 casos no estuvo referida como un factor negativo de la propia cumbre o de sus patrocinadores sino como un elemento más que debería evitarse.

Otros parámetros que se tuvieron en cuenta se basaron en si los contenidos eran digitales nativos o aprovechados de los medios matriz. Esta política de reutilización de contenidos en diferentes canales del mismo medio se identifica como crossmedia (Siapera \&Veglis, 2012) ypuededenotar elinterés por ofrecerenfoques específicos según el canal o, en sentido contrario, un modelo de aprovechamiento de costes $^{18}$. En línea con esta variable, el análisis de contenido observó si las noticias publicadas eran originales o réplicas de teletipos de agencias de noticias.

Dendograma 1. Dendrograma organizado con las categorías de los contenidos de medios digitales tomando como origen la relación del término greenwashing con la COP25

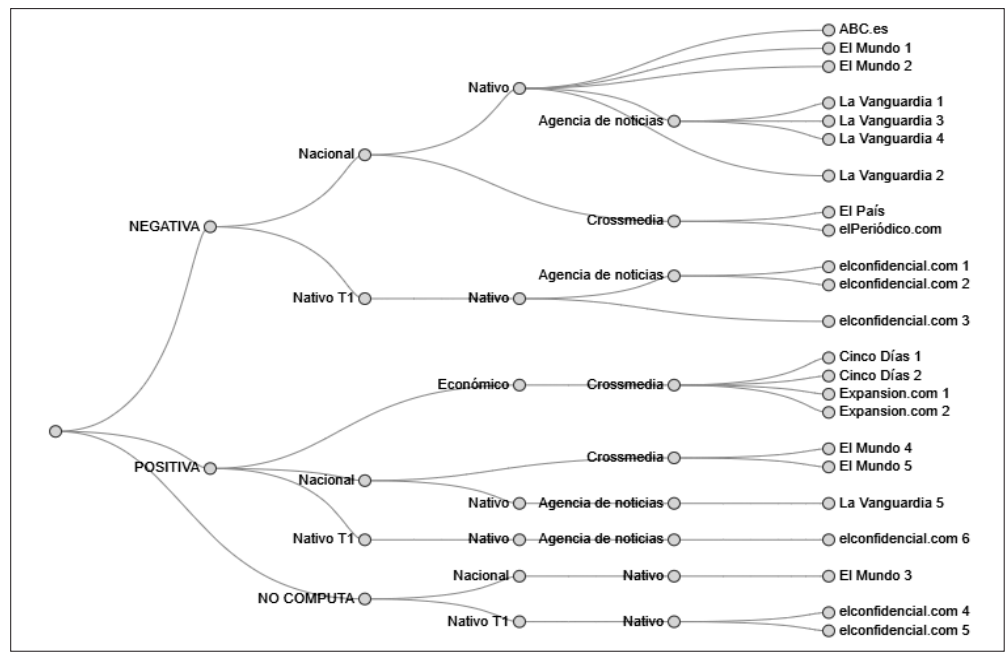

Fuente: elaboración propia.

18 La labor periodística se reutiliza con los mínimos cambios posibles para cada uno de los soportes del medio. 
Como puede observarse, en la figura gráfica anterior se representan las características de los contenidos analizados sin advertir ninguna constante de correlaciones entre la dualidad positivo/negativo y otras variables, como si se tratase de una noticia digital nativa, con origen en nota de prensa, de un medio de comunicación en particular, etc. Sin embargo, aunque según estos datos puede afirmarse la indiferencia en las características como condicionante, el caso de los medios consideradoseconómicos constituye una excepción. Esta tipología de medios lanzó contenidos sólo de tipo positivo con la particularidad de que todos estos venían reutilizados del medio matriz en papel (dinámica crossmedia).

Por otro lado, el análisis en detalle indica que los contenidos negativos estuvieron marcados temáticamente por acciones reivindicativas en reclamo de mayor acción en favor del clima y el cese del greenwashing (8 contenidos de $\operatorname{los} 12$ totales) ${ }^{19}$. Estas acciones denotaron la ausencia de instituciones u organizaciones como productores de los mensajes, en coherencia con las acciones de comunicación de patrocinio y la dualidad público/privada en la organización del evento.

En todo caso los resultados obtenidos no mostraron un patrón homogéneo en el resto de las variables, salvo la ya mencionada publicación de contenidos positivos y crossmedia por parte de los medios digitales de prensa económica. Esto es debido a que no se observó dependencia entre relación (positiva, negativa), tipología (nacional, Nativo1) y formato de los contenidos (nativo, teletipo, crossmedia).

\subsection{Análisis de la prensa tradicional}

El análisis de contenido sobre la relación del concepto greenwashing con la cumbre indicó que en prensa tradicional 14 artículos consideraron que existía una relación de tipo negativo: la Conferencia era espacio para ellavado de imagen verde de diferentes instituciones. En cambio 11 contenidos consideraron de forma positivala relación entrela COP25y el greenwashing: la cumbre permitió sacar a la luz este fenómeno y pasar de las percepciones a las realidades ${ }^{20}$.

Proporcionalmente el conjunto de contenidos que mencionaron el concepto de greenwashing de forma negativa se reveló, en sintonía con los medios digitales, muy equilibrado en relación a los artículos que generaron una vinculación positiva. El total de artículos de prensa tradicional que relacionaron positivamente la cumbre con el fenómeno greenwashing como estrategia a erradicar llegó al 56\%, mientras que el 44\% de artículos mostraron un nexo negativo.

19 Aunque se produzca cierto equilibrio entre números de contenidos con orientación positiva y número de con tenidos con orientación negativa, los datos de audiencia de medios digitales señalaron que la relación positiva alcanzó a 9.667.515 usuarios (32\%) en comparación a 20.905.219 usuarios (68\%) que potencialmente accedieron a contenidos relacionados negativamente. Esta desviación en la proporción de contenidos es resultado de que los medios considerados económicos alcanzan como media una audiencia sensiblemente inferior a los medios digitales que tienen como origen prensa generalista, pese a que esta audiencia sea un público mucho más específico.

20 Uno de los contenidos no se computó porque, aunque concepto y cumbre formaban parte del mismo artículo, no había relación entre ambos al tratarse de un decálogo de términos. 
Similar equilibrio aparece en el caso de la prensa tradicional si se calcula la audiencia en términos de difusión de los medios, medida que representa el total de ejemplares adquiridos realmente por los lectores. Pese a que los medios nacionales y económicos ofrecen por regla general más audiencia potencial que los regionales ${ }^{21}$, este desequilibrio no alteró significativamente los resultados obtenidos en el acumulado del total de contenidos. La audiencia potencial que estuvo expuesta a la vinculación negativa entre la COP25 y el fenómeno greenwashing fue de un $48 \%$ en comparación al 52\% que advirtió una relación positiva.

Por lo tanto, la observación de tipo cuantitativo de los datos extraídos por análisis de contenido ${ }^{22}$ no indica grandes variaciones desde una perspectiva exploratoria. Sin embargo, una observación en detalle basada en la tipología de los medios que sirvieron de soporte a una vinculación negativa o positiva permite la aparición de un fenómeno de tipo cualitativo relacionado con la categorización de los medios.

Dendograma 2. Dendrograma organizado con las categorías de los contenidos de prensa tradicional tomando como origen la relación del término greenwashing con la COP25

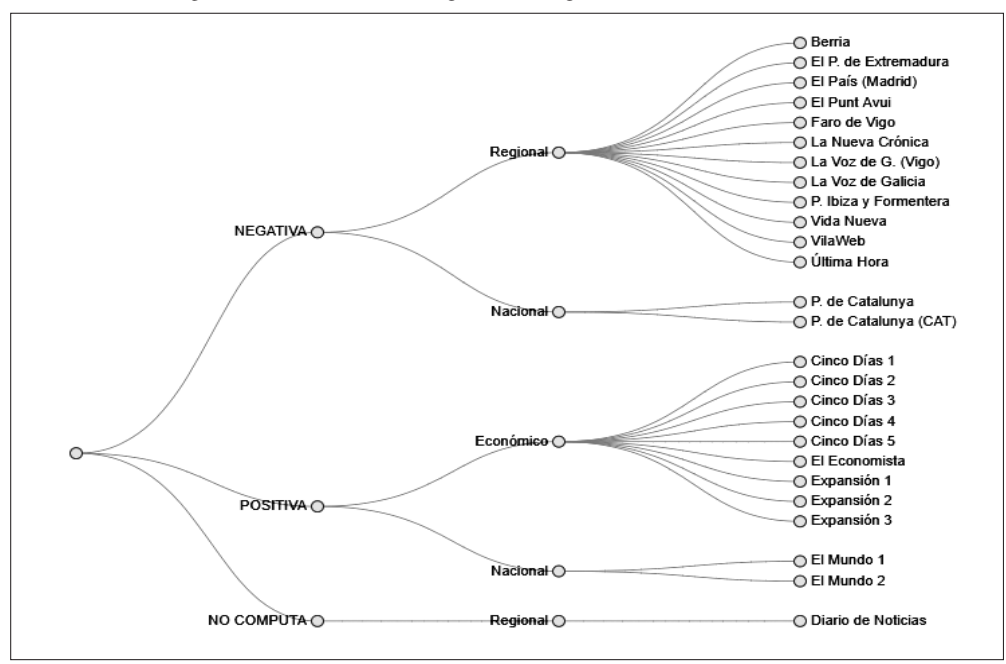

Fuente: elaboración propia.

Como puede verse en la representación gráfica, existe una directriz homogénea que conecta directamente las menciones positivas al greenwashing dentro de la COP25 con los medios de tirada nacional generalistas y económicos. Estos medios trasladaron la alerta contra la problemática de la estética medioambiental usando principalmente como vehículo las declaraciones de

21 Laúnicapublicación nodiaria queentróenlainvestigaciónfuela revista Vida Nueva, deperiodicidadsemanal(salecada sábado), que se categorizó como regional para evitar generar una sección nueva que no aportaría hallazgos relevantes.

22 Audiencias mediante recuperación dela información y vinculación positiva o negativa mediante análisis humano. 
participantes de la cumbre ${ }^{23}$ y señalando que ese era el escenario para convertir los hechos en palabras ${ }^{24}$.

Por el contrario, existe una clara hegemonía de los medios regionales en la interpretación negativa y contraria a la comunicación estratégica desarrollada por los organizadores. Estos medios monopolizaron casi totalmentelas menciones en que se criticó a la COP25 como escenario para el greenwashing. Una única discordancia supone la noticia del medio nacional El periódico de Catalunya ${ }^{25}$, en la que se lamentaba la política medioambiental de la ciudad de Madrid. Sin embargo, resulta de gran relevancia tener en cuenta que esta noticia no estaba firmada por el medio o por un periodista, sino que se trató de una carta de unalectora del medio enviada al director del periódico. En estos casos, tal como comenta Pastor (2010), estaríamos ante una comunicación de tipo privada que "el director de una publicación extiende a un público de personas afines porque cree que pueden llegar a interesar" (p. 66). En este caso, por tanto, la narración trasladada por los medios tiene como portavoza unalectorayno al propio medio (que ejerce de gatekeeper) o a la traslación de la opinión del responsable de una institución.

Por esta razón, debido al condicionante mostrado, es posible afirmar que el tratamiento del concepto greenwashing sólo tuvo una visión desfavorable en medios de alcance regional con una importancia cuantitativa equilibrada en términos de número de contenidos o de audiencia ( $48 \%$ del total desagregando a El periódico de Catalunya). En todo caso, sigue tratándose de una exposición no deseada a un mensaje que contradice completamente los esfuerzos de relaciones públicas para mejorar la imagen de las organizaciones. Este efecto no sólo es un outcome no deseado en el que los medios ejercen de correa de transmisión como stakeholders, sino que es directamente proporcional a una acción de comunicación estratégica (a más inversión en fomentar la imagen pública en la COP25, mayor traslación por parte de algunos medios de su visión negativa y mayor opinión contraria).

\subsection{Perspectiva cualitativa: medios como stakeholders y público objetivo}

Como ha podido detallarse existe, desde un punto de vista cuantitativo, cierta equivalencia entre relación positiva o negativa del greenwashing que sería susceptible de mantenerse en el tiempo tanto en número de impactos como en audiencias expuestas. Sin embargo, la observación de la dimensión cualitativa puede ofrecer una valoración más concreta sobre la verdadera afectación de las críticas a un supuesto disfraz sostenible de las instituciones.

Tal y como se ha comentado, los medios regionales trasladaron de forma

23 Como la declaración de José Manual Entrecanales, Presidente Ejecutivo de Acciona (empresa patrocinadora de la cumbre) o la de Nadia Calviño, Ministra de Economía del gobierno que organizó la cumbre.

24 El lema de la Conferencia sobre el Cambio Climático fue: "Es tiempo de actuar".

25 Ediciones en castellano y catalán. 
compacta una visión negativa de la COP25 con respecto a las políticas de greenwashing; por el contrario, los medios nacionales y económicos expresaron una dinámica positiva. La indefinición mostrada por la medición cuantitativa se desvanece cuando se comprende quién es el público objetivo de cada una de las categorías de medios que optaron por una u otra opción. Como detalla Capriotti (2007), la existencia de los públicos en el campo de las relaciones públicas se encuentra vinculada con la "interacción específica que haya establecido la organización con su entorno" (pp.76y77). Sibien una evaluación de tipo cuantitativo siempre debería señalar un éxito a nivel proporcional de las acciones de comunicación estratégica respecto las reacciones perjudiciales que puedan producirse, una perspectiva cualitativa debería enfocar en la importancia de los públicos receptores y posibles stakeholders de las organizaciones.

Según los datos de esta investigación la afectación del outcome negativo referido al greenwashing quedó circunscrita a los medios regionales de prensa y las interpretaciones positivas fueron reconocidas con cierta exclusividad por los medios considerados económicos tanto en su versión papel como en su versión digital. Este hallazgo es del máximo interés si se coloca en comunión con los públicos objetivos de estos medios, que serían lectores de nivel adquisitivo alto con importante presencia de profesionales directivos y, por lo tanto, potencialmente inversores o colaboradores a nivel empresarial ${ }^{26}$.

A su vez la propia naturaleza del fenómeno greenwashing acentúa la importancia del target tipo de estas publicaciones, pues como argumentan estudios previos son los accionistas y los socios a nivel de negocio los stakeholders que disponen de la capacidad de forzar cambios reales en la política medioambiental de las organizaciones (Testa, Boiral \& Iraldo, 2018). De allí que la opinión de los medios económicos, como stakeholders en sí mismos, es significativa, y un cambio en su visión (desde una cobertura promovida hasta una versión divergente) afecta decisivamente a la imagen de las organizaciones ante los públicos clave, que en este caso serían potenciales stakeholders legitimados para actuar. Como indica Theaker (2016), los profesionales en el campo de las relaciones públicas "pretenden influir en los medios de comunicación, en parte debido a su alcance, pero también por su credibilidad ante el público objetivo con el que desean conectar" (p. 277).

No obstante, según los resultados obtenidos en esta investigación, esta no sería la situación existente. Los mismos permiten deducir la presencia de un impacto atenuado (a nivel cuantitativo) y completamente limitado (a nivel cualitativo), tal como puede observarse en el siguiente gráfico.

26 Los datos demográficos de consumo de prensa en versión papel señalan que el diario Expansión es el medio económico más leído con una audiencia compuesta en un $89 \%$ por clase media-alta (véase: http://www.unidadeditorial.es/publicidad/images/prensa/expansion/mediabook/Expansion_Ediciones.pdf. En segundo lugar, e diario Cinco Días tiene un $27 \%$ de audiencia categorizada como rango directivo (véase: https://www.prisabs.com/ es/info/cinco-dias) y el $42 \%$ de la audiencia del medio El Economista es de clase social alta (véase: http://estatico. cmvocento.com/download/products/argumentario-el-economista/argumentario-318.pdf). 
Diagrama 2. Diagrama con el marco de evaluación y la variante de análisis del outcome no intencionado aplicado en esta investigación

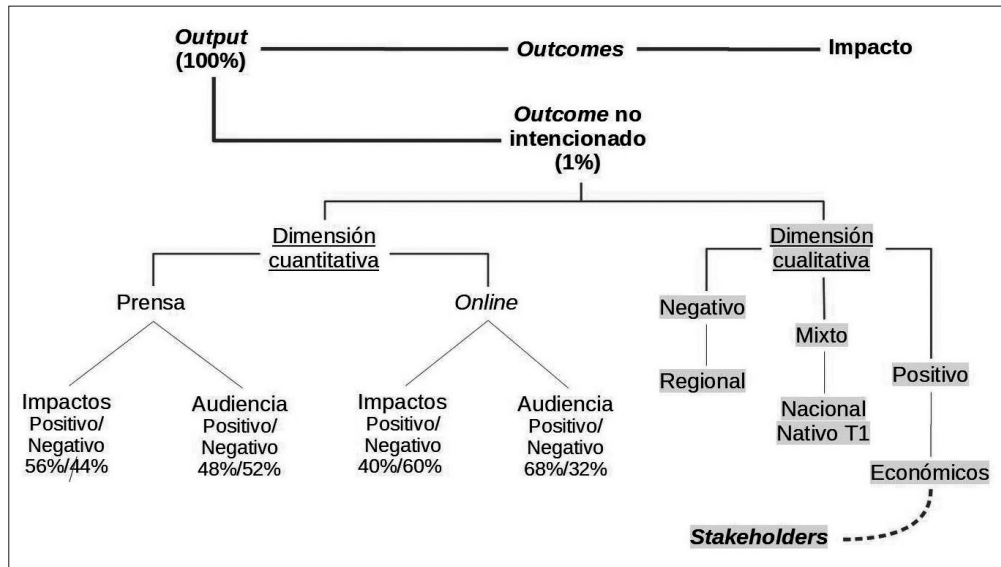

Fuente: elaboración propia.

Esta aproximación integradora, elaborada mediante variables cuantitativas y cualitativas, aporta, por un lado, lo que Macnamara y Greg (2018) demandan como un enfoque "holístico e integral de la evaluación que considere todos los resultados eimpactos dela comunicación como parte dela estrategia futura" (p. 481), como fuente de información y como modelo de aprendizaje. A su vez y en una dimensión a más corto plazo puede conducir a la formulación de contramedidas que minimicen la expansión del outcome negativo evaluado. En el caso que nos ocupa, y tomando como referentela importancia de los medios de comunicación como stakeholder que es a su vez generador de posicionamiento ante el resto de los públicos, subyace la relevancia de las relaciones con los medios entendidas como el conjunto de interacciones que se producen entre una organización y los responsables de los medios de comunicación y periodistas (Brunner, 2019) ${ }^{27}$. Relación que se desarrollará siempre desde una actitud de colaboración (Castillo Esparcia, 2010) y de generación de valor tanto para las organizaciones como para los periodistas (Xifra, 2007).

\section{CONCLUSIONES}

Esta investigación ha pretendido observar de una forma amplia e integradora la traslación por parte de los medios del fenómeno greenwashing dentro de la celebración de la COP25 en Madrid. Esta situación representaría un resultado (outcome) no deseado y directamente contraproducente respecto a

27 Según Brunner (2019) estas actividades podrían listarse en: generación de contenidos informativos, monitorización de la cobertura en los medios, resolución de consultas de los periodistas, obtención de cobertura favorable y reducción de la cobertura negativa en relación con la organización. 
la estrategia de patrocinio de la cumbre y de recepción de cobertura positiva relacionada con la misma. Si un objetivo general de los patrocinadores era mejorar la percepción de la imagen de marca y relacionarla unívocamente con el respeto ambiental, resulta muy significativa esta línea discursiva paralela y antagónica que debe ser analizada desde diferentes puntos de vista.

Tal como se ha mencionado en el marco teórico, la actividad de la comunicación estratégica debería reconocer que esta puede desencadenar, mediante una lógica de acción y reacción, un efecto desfavorable para los diferentes públicos o grupos de interés relacionados con la organización. El presente ha sido un estudio de caso paradigmático en el que la actividad de relaciones públicas ha sido generadora de posibles efectos negativos de forma proporcional, cual vasos comunicantes.

El análisis de contenido y la observación de las características de las noticias y posts incorporados a la investigación señalan un impacto significativo en términos de audiencia. Esta sería una evaluación de tipo cuantitativo que puede contraponerse con las audiencias totales generadas por la cobertura de la cumbre. No obstante, hay dos cuestiones relevantes más allá de la confrontación de volúmenes potenciales.

En primer lugar, que los outcomes representan la exigencia de ir más allá de la medición de coberturas para indicar la medición de cambios reales. Ese cambio efectivo, en este caso negativo, se produce cuando los medios se separan de la línea argumental de la cumbre (tanto pagada como no pagada) e inician un tratamiento alternativo y claramente contradictorio.

En segundo orden de importancia, se evidencia que una elevada movilización de exposición al mensaje ${ }^{28}$, en este caso negativa, no confirma el acceso a los públicos clave. Como se ha mencionado anteriormente, el fenómeno del greenwashing puede ser detonante para que inversores y empresas colaboradoras fuercen cambios directos en la gestión de las compañías. Por lo tanto, es conveniente ir más allá de la mera cuantificación de impactos o de las características de estos: resulta necesario un análisis en detalle de todos los parámetros disponibles dado que pueden mostrar los riesgos concretos sobre los que se debe actuar. Tomando a este trabajo como referente, el peligro más acuciante sería que los medios fueran vehículos para que otros stakeholders reorienten la relación existente con la organización.

Finalmente, este trabajo espera ser un pequeño aporte a la escasamente estudiada explotación de la teoría de los stakeholders enfatizando en la relación entre una organización y sus públicos (Capriotti, 2007). No en vano la comunicación estratégica es tal por la orientación a unos objetivos que podrían no alcanzarse cuando la vinculación con los públicos no es la deseada, especialmente si estos tienen poder para influir en la organización.

28 En términos de audiencia potencial absoluta y no en términos de audiencia relativa respecto a la cobertura de la cumbre. 


\section{REFERENCIAS}

Argenti, P. A., Howell, R. A. \& Beck, K. A. (2005). The strategic communication imperative. MITSloanmanagementreview, 46(3),83-89. Availableon:https://www.researchgate. net/publication/252084158_The_Strategic_Communication_Imperative

Austin, E. W. \& Pinkleton, B. E. (2015). Strategic public relations management: Planning and managing effective communication campaigns. London: Routledge. DOI: https://doi.org/10.4324/9781315754833

Bartholomew, D. (2016). Metrics man: It doesn't count unless you can count it. Nueva York: Business Expert Press

Buhmann, A. \& Brønn, P. S. (2018). Applying Ajzen's theory of planned behavior to predict practitioners' intentions to measure and evaluate communication outcomes. Corporate Communications: An International Journal, 23(3), 377-391. DOI: https://doi.org/10.1108/ccij-11-2017-0107

Brunner, B. R. (2019). Public Relations Theory: Application and Understanding. Nueva Jersey: Wiley-Blackwell.

Capriotti, P. (2007). El concepto de relación como fundamento de la naturaleza de los públicos en Relaciones Públicas. Sphera Pública, (7), 65-80. Recuperado de: https://www.redalyc.org/pdf/297/29720421005.pdf

Castillo Esparcia, A. (2010). Introducción a las relaciones públicas. España: Instituto de Investigación en Relaciones Públicas.

DeJong,M.D.,Harkink, K.M.\&Barth,S.(2018).Makinggreen stuff?Effects of corporate greenwashing on consumers. Journal of business and technical communication, 32(1), 77-112. DOI: https://doi.org/10.1177/1050651917729863

Delmas, M. A. \& Burbano, V. C. (2011). The drivers of greenwashing. California management review, 54(1), pp. 64-87. DOI: https://doi.org/10.1525/ cmr.2011.54.1.64

Freeman, R. E. \& Reed, D. L. (1983). Stockholders and stakeholders: A new perspective on corporate governance. California management review, 25(3), 88-106. DOI: https://doi.org/10.2307/41165018

Gallicano, T. D. (2011). A critical analysis of greenwashing claims. Public Relations Journal, 5(3), 1-21. Available on: https://www.researchgate.net/ publication/305438010_A_Critical_Analysis_of_Greenwashing_Claims

Hallahan, K. (2013). Communication management. In R. L. Heath (Ed.), Encyclopedia of public relations (pp. 161-164). Thousand Oaks: Sage

Hallahan, K., Holtzhausen, D., Van Ruler, B., Verčič, D. \& Sriramesh, K. (2007). Defining Strategic Communication. International Journal of Strategic Communication, 1 , 3-35. DOI: https://doi.org/10.1080/15531180701285244. 
Krippendorff, K. (2018). Content analysis: An introduction to itsmethodology. Thousand Oaks: Sage

Lindenmann, W. K. (1997). Setting minimum standards for measuring public relations effectiveness. Public Relations Review, 23(4), 391-402. DOI: https://doi. org/10.1016/s0363-8111(97)90053-9

Lindenmann, W.K. (2003). Guidelinesformeasuringthe Effectiveness of PR Programsand Activities. Gainesville: Institute for Public Relations.

L'Etang, J. \& Pieczka, M. (2006). Public relations: Critical debates and contemporary practice. Nueva York: Routledge.

Lyon, T. P. \& Montgomery, A. W. (2015). The means and end of greenwash. Organization «Environment, 28(2),223-249.DOI:https://doi.org/10.1177/1086026615575332

Macnamara, J. (2014). The development of international standards for measurement and evaluation of public relations and corporate communication: A review. Report for the Australian Centre for Public Communication, University of Technology Sydney, November. Available on: https://www.uts.edu.au/sites/default/files/acpcpr-measurement-and-evaluation-review.pdf

Macnamara, J. (2017). Evaluating public communication: Exploring new models, standards, and best practice. Nueva York: Routledge.

Macnamara,J.(2018a). A review of new evaluation models for strategic communication: Progress and gaps. International Journal of Strategic Communication, 12(2), 180195. DOI: https://doi.org/10.1080/1553118x.2018.1428978

Macnamara, J. (2018b). 11 Content Analysis. In P. M. Napoli (Ed.), Mediated Communication (pp. 191-212). Berlin, Boston: De Gruyter.

Macnamara, J. (2018c). Evaluating public communication: Exploring new models, standards, and best practice. London: Routledge.

Macnamara, J. \& Gregory, A. (2018). Expanding evaluation to progress strategic communication: Beyond message tracking to openlistening. International Journal of Strategic Communication, 12(4), 469-486. DOI: https://doi.org/10.1080/1553 118x.2018.1450255

Madroñero, G. \& Capriotti, P. (2018). La etapa de la Estrategia de Comunicación en el proceso de planificación de la comunicación y las relaciones públicas. Revista InternacionaldeRelacionesPúblicas, 8(16),171-186.DOI:https://doi.org/10.5783/ rirp-16-2018-10-171-186

Medina, A. (1 de diciembre de 2019). Acciona, Endesa, Engie e Iberdrola, los grandes patrocinadores de la COP25. Expansión. Recuperado de: https://www.expansion. com/economia/2019/12/01/5de3f23e468aeb451c8b463d.html 
Michaelson, D. \& Stacks, D. W. (2011). Standardization in public relations measurement and evaluation. Public Relations Journal, 5(2). Available on: https://prjournal. instituteforpr.org/wp-content/uploads/Standardization.pdf

Neuendorf, K. A. (2017). The content analysis guidebook. Thousand Oaks: Sage.

Nyilasy, G., Gangadharbatla, H. \& Paladino, A. (2014). Perceived greenwashing: The interactive effects of green advertising and corporate environmental performance on consumer reactions. Journal of Business Ethics, 125(4), 693-707. DOI: https:// doi.org/10.1007/s10551-013-1944-3

Pastor, L. (2010). Teoría de las cartas al director. La gestión periodística del público I. Barcelona: Editorial UOC.

Pieczka, M. (2019). Looking back and going forward: The concept of the public in public relations theory. Public Relations Inquiry, 8(3), 225-244. DOI: https://doi. org/10.1177/2046147x19870269

Place, K. R. (2015). Exploring the role of ethics in public relations program evaluation. Journal of Public Relations Research, 27(2), 118-135. DOI: https://doi.org/10.108 0/1062726x.2014.976825

Rawlins, B. L. (2006). Prioritizing stakeholders for public relations. Institute for publicrelations. Available on: https://instituteforpr.org/wp-content/ uploads/2006_Stakeholders_1.pdf

Scholes, E. \& Clutterbuck, D. (1998). Communication with stakeholders: An integrated approach. Long range planning, 31(2), 227-238. DOI: https://doi.org/10.1016/ s0024-6301(98)00007-7

Schriner, M., Swenson, R. \& Gilkerson, N. (2017). Outputs or Outcomes? Assessing Public Relations Evaluation Practices In Award-Winning PR Campaigns. Public Relations Journal, 11(1). Available on: https://epublications.marquette.edu/cgi/ viewcontent.cgi? article $=1481 \&$ context $=$ comm_fac

Siapera, E. \& Veglis, A. (2012). The Handbook of Global Online Journalism. Malden: Wiley-Blackwel.

Smith, H. J. (2003). The Shareholders vs. Stakeholders Debate. MIT Sloan Management Review, 44(4), 85-90. Available on: https://sloanreview.mit.edu/wp-content/ uploads/2003/07/b966946aae.pdf

Söderqvist, R. (2017). Explaining Outcome-The role of utilizing measurement insights for planning and reporting. A quantitative analysis of Swedish communicators. Available on:http://lup.lub.lu.se/luur/download?func=downloadFile\&recordOI $\mathrm{d}=8927925$ \&fileOId $=8927929$

Swenson, R., Gilkerson, N., Likely, F., Anderson, F.W. \& Ziviani M. (2019). Insights from Industry Leaders. International Journal of Strategic Communication. DOI: https:// doi.org/10.1080/1553118x.2018.1533555 
Testa, F., Boiral, O. \& Iraldo, F. (2018). Internalization of environmental practices and institutional complexity: can stakeholders pressures encourage greenwashing? Journal of Business Ethics, 147(2), 287-307. DOI: https://doi.org/10.1007/s10551015-2960-2

Theaker, A. (2016). The public relations handbook. London, New York: Routledge.

Villarreal, A. (3 de diciembre de 2019). El 'greenwashing' del Ibex 35 con la Cumbre: chapa y pintura verde para grandes empresas. El Confidencial. Available on: https://www.elconfidencial.com/empresas/2019-12-03/cop25-greenwashingempresas-ibex-35_2361780/

Watson, T. (2013). Advertising value equivalence-PR's orphan metric. Public Relations Review, 39(2), 139-146. DOI: https://doi.org/10.1016/j.pubrev.2012.11.001

Watson, T. \& Noble, P. (2014). The evolution of evaluation. Communication Director, (3), 44-47. Available on: https://www.researchgate.net/profile/ Tom_Watson5/publication/269697336_The_evolution_of_evaluation/ links/5492d0ec0cf2302e1d0742ba/The-evolution-of-evaluation.pdf

Xifra, J. (2007). Técnicas de las relaciones públicas. Barcelona: Editorial UOC.

* Contribución: $100 \%$ del autor.

\section{(cc) BY}

Artículo publicado en acceso abierto bajo la Licencia Creative Commons - Attribution 4.0 International (CC BY 4.0).

\section{IDENTIFICACIÓN DEL AUTOR}

Francisco L. López del Castillo Wilderbeek. Doctor en Comunicación por la Universitat Pompeu Fabra (UPF), España. Documentation Expert en la empresa de análisis de medios de comunicación REBOLD, España, y miembro del grupo de investigación MEDIUM en la UPF. Además del doctorado, ha cursado estudios de Derecho, Comunicación Audiovisualy el Máster de Sociedad dela Información del Conocimiento en la Universitat Oberta de Catalunya (UOC), España. Su producción científica está centrada en el ámbito de la evaluación de las acciones de comunicación como la aplicación del paradigma psicofisiológico o de la perspectiva semiótica. Entre sus últimos trabajos pueden mencionarse: "Outcomes en la evaluación de las relaciones públicas, la aproximación semiótica" (2019, Revista Internacional de Relaciones Públicas); "La psicofisiología como herramienta parala evaluación de la publicity” (2019, Estrategas. Investigación en Comunicación).

\section{REGISTRO BIBLIOGRÁFICO}

López del Castillo, F. L. (enero-junio, 2020). Stakeholders y outcomes no intencionados en la comunicación estratégica. InMediaciones de la Comunicación, 15(1), 133-153. 